\title{
Detección y diagnóstico de fallas en sistemas complejos de manufactura empleando técnicas de softcomputing
}

\author{
Juan Pablo Nieto González \\ Universidad Autónoma de Coahuila, \\ Coahuila, México \\ juan.nieto@uadec.edu.mx
}

\begin{abstract}
Resumen. La principal meta de un sistema de detección y diagnóstico de fallas en sistemas de manufactura es prevenir un paro no deseado de la línea de producción y su correspondiente costo. Los modernos sistemas de manufactura son un dominio bastante complejo debido a todas las variables físicas involucradas y a sus correlaciones. Lo cual representa una tarea bastante retadora para los sistemas de monitoreo. El presente artículo muestra un sistema de detección y diagnóstico de fallas basado en los datos históricos del proceso. La propuesta esta compuesta por dos fases. En la primera fase aprende el comportamiento de la operación normal del sistema utilizando una red neuronal autoasociativa (RNAA), la cual lleva a cabo el proceso de detección. En la segunda fase se da el diagnóstico final empleando una máquina de soporte vectorial multiclase (MSV), la cual clasifica el tipo de falla presente y proporciona su tiempo de ocurrencia.
\end{abstract}

Palabras clave: Detección de fallas, diagnóstico de fallas, sistemas complejos, ed neuronal autoasociativa, máquina de soporte vectorial.

\section{Fault Detection and Diagnostics in Complex Manufacturing Systems using Softcomputing Techniques}

\begin{abstract}
The main goal of a fault detection and diagnosis of manufacturing systems is to prevent an undesired stop of the production line and its corresponding cost. Modern manufacturing systems are a very complex domain due to all the physical variables involved and their correlations. This represent a very challenging task for monitoring systems. The present article shows a fault detection and diagnosis system based on the history data process. The proposal is composed by two phases. In the first phase it learns the normal operation behavior of the system using an autoassociative neural network which carries out the detection process. In the second phase the final diagnosis is given using
\end{abstract}


a multiclass support vector machine, which classifies the type of fault present and gives its time of occurrence.

Keywords: Fault detection, fault diagnostics, complex systems, autoassociative neural network, support vector machine.

\section{Introducción}

Desde el comienzo de la era de las máquinas, los humanos han estado interesados en sus condiciones de trabajo. Durante casi toda la historia de la humanidad, la única manera de aprender acerca de las malas operaciones de los sistemas y sus correspondientes subsistemas, ha sido mediante el empleo de solamente los cinco sentidos. Posteriormente se da la implementación de los sensores, que son elementos destinados a tomar lecturas y son utilizados para dar a los humanos el estado de ciertas variables físicas y de ésta manera detectar si un sistema o proceso está trabajando adecuadamente o no. Con el gran avance tecnológico los procesos se han vuelto cada vez más complejos, por lo cual su monitoreo es bastante importante para mejorar su desempeño, eficiencia y asegurar la calidad del producto terminado. Es entonces, que el análisis y diagnóstico de fallas puede ayudar a evitar pérdidas de producción y accidentes, que ponen en riesgo la salud y vida de los operadores y daño al equipo. El diagnóstico de fallas de los sistemas de ingeniería esta relacionado a la detección de fallas en máquinas complejas detectando patrones específicos de comportamiento en los datos observados. Un moderno sistema de manufactura es un ejemplo de tal sistema de ingeniería complejo dónde existen un gran número de sensores, controladores y módulos de cómputo que recolectan una gran cantidad de señales. Basado en estos hechos y considerando que los modernos sistemas de manufactura industrial, ya sean de una planta industrial completa o una sola máquina, son sistemas de gran escala y de ellos se pueden extraer una gran cantidad de datos, es que se propone una metodología de monitoreo y diagnóstico de fallas que emplea solamente datos históricos del proceso combinando una red neuronal autoasociativa (RNAA) y una máquina de soporte vectorial multiclase (MSV). La metodología se aplica a los motores de una banda transportadora mostrando resultados prometedores. La organización del artículo es la siguiente. La sección 2 revisa el estado del arte. La sección 3 da los preliminares matemáticos de la RNAA y de la MSV. La sección 4 proporciona la descripción general de la propuesta. La sección 5 muestra un caso de estudio. Finalmente la sección 6 da la conclusión del artículo.

\section{Estado del arte}

El incremento en los requerimientos para alcanzar un desempeño más confiable de sistemas complejos ha motivado el desarrollo de esquemas de diagnóstico de fallas que puedan realizar un diagnóstico más preciso. En el presente 
artículo se propone una metodología para el diagnóstico de sistemas complejos. La propuesta fue aplicada al monitoreo de motores eléctricos de una celda de manufactura. Desde el punto de vista de seguridad y confiabilidad de los sistemas eléctricos, es necesario tener un oportuno diagnóstico de fallas que pueda detectar, aislar y diagnósticar fallas y de avisar a los operadores del sistema para tomar las correspondientes acciones correctivas. Durante un disturbio, hay un gran número de eventos relacionados a las fallas, haciendo que el diagnóstico y la decisión de tomar acciones correctivas se torne una tarea difícil. En este dominio, la necesidad de desarrollar técnicas más poderosas ha sido reconocida, y las técnicas híbridas que combinan varios métodos de razonamiento se han empezado a emplear. [1] considera la configuración de elementos automáticos en los modernos sistemas de potencia eléctrica, tales como relevadores de protección y de recierre automáticos para mejorar un modelo analítico y de optimización para el diagnóstico de fallas de sistemas de potencia. De acuerdo al principio de protección de los relevadores, el diagnóstico de fallas es expresado como un problema de programación entera y resuelto por el algoritmo genético de búsqueda Tabú. [2] presenta una metodología que utiliza redes neuronales integradas con varias técnicas estadísticas. Entre las herramientas numéricas y estadísticas utiliza análisis de Fourier, valores RMS, valores de sesgo y de curtosis así como componentes simétricas para la detección y la identificación de las fallas es llevada a cabo mediante una red neuronal perceptrón multicapa. [3] emplea las formas de onda de corriente y les aplican un análisis en frecuencia y onduletas para generar un algoritmo de formación de grupos para clasificar si el sistema se encuentra en modo de falla o no. [4] utiliza lógica difusa para la detección de fallas en motores de inducción. La propuesta esta compuesta por dos etapas. Extracción de razgos y la implementación de un sistema difuso. Se utilizan las magnitudes de las corrientes trifásicas de un motor para detectar y caracterizar las fallas. [5] propone un método compuesto de dos fases: En la primera fase una red neuronal probabilística es entrenada con los eigenvalores de los datos de voltaje obtenidos en operación normal así como con fallas simétricas y asimétricas. La segunda fase emplea una comparación entre las muestras para detectar y localizar la presencia de una falla. [6] presenta una metodología de diagnóstico basada en un sistema difuso que interpreta las señales provenientes de los sensores de corriente de un motor de inducción. [7] encuentra señales de entropía y la curtosis, para posteriormente alimentarlas como entradas de una red neuronal y así llevar a cabo la clasificación de las fallas. [8] utiliza lecturas de la corriente de fase solo durante el primer cuarto de ciclo empleando un método que combina componentes simétricas con un análisis de componentes principales para identificar y clasificar una falla. [9] puede analizar fallas que ocurren entre dos líneas equipadas con unidades de medición. El primer paso de la metodología es detectar la presencia de una falla en tiempo real. Luego, el método de componentes simétricas es utilizado para convertir señales trifásicas en tres conjuntos de componentes independientes, los cuales son las secuencias positiva, negativa y cero. [10] propone una red bayesiana y minería de datos para diagnosticar fallas en una red eléctrica. El estatus de la información de las 
protecciones es tomado como atributos condicionales y la región de falla como un atributo de decisión. [11] relaciona la incertidumbre asociada a un evento con una distribución de probabilidad. Utiliza un algoritmo difuso aplicado a cada valor de entropía para realizar la identificación de una falla. [12] propone una metodología capaz de localizar nodos de un sistema eléctrico en modo de falla. La metodología está compuesta por dos fases: en la primer fase una red neuronal probabilística se entrena con los eigenvalores de voltaje para dar una clasificación del tipo de falla presente. En la segunda fase se utiliza un ANFIS para dar el diagnóstico final.

\section{Preliminares}

\subsection{Red neuronal autoasociativa (RNAA)}

[13] propuso una RNAA utilizada como un método de Análisis de Componentes Principales No-Lineales (ACPNL) para identificar y remover correlaciones entre las variables de un problema como una ayuda para reducir la dimensionalidad, visualización de datos, y análisis exploratorio de datos. ACPNL opera entrenando una red neuronal tipo feed-forward para llevar a cabo un mapeo idéntico de las entradas de la red y reproducirlas en la capa de salida. La red contiene una capa interna que actúa como un cuello de botella (la capa contiene una menor cantidad de neuronas que las capas de entrada o de salida), lo cual obliga a la red a desarrollar una representación compacta de los datos de entrada. La RNAA está compuesta por cinco capas. La Figura 1 muestra la arquitectura de la RNAA.

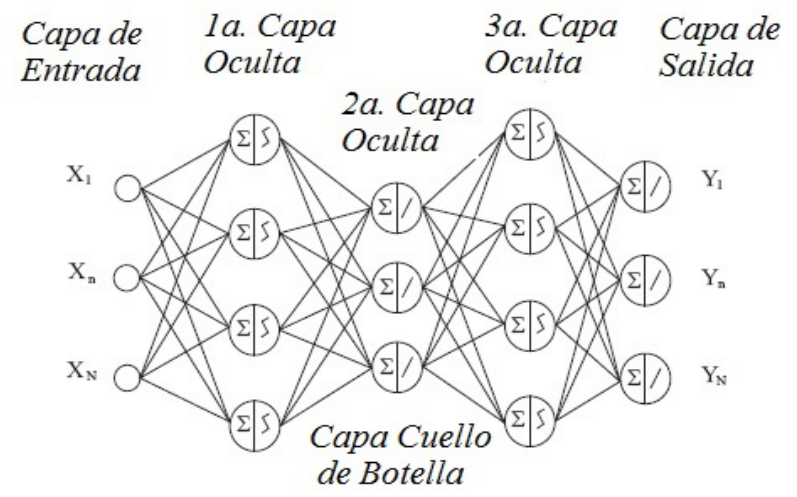

Fig. 1. Arquitectura de una RNAA

Esta RNAA tiene una capa de entrada y una capa de salida, cada una con $N$ neuronas y tres capas ocultas con $H_{1}, H_{2}$, y $H_{3}$ neuronas respectivamente. Cuando una observación $x$ es presentada en la entrada de la red, la ecuación de 
la neurona de salida de la $l^{\text {th }}$ capa es función de las neuronas en la $(l-1)^{t h}$ capa, dada por la ecuación 1 y mostrada en la Figura 2.

$$
y_{j}=f\left(\sum_{i=1}^{n(l-1)} w_{i, j}^{(l)} y_{i}^{l-1)}\right) l=1, \ldots, 4,
$$

donde $y_{j}^{(0)}=x_{n}, n=1, \ldots, N$ representa las componentes del vector de observaciones a la entrada de la red; $y_{n}^{(4)}, n=1, \ldots, N$, representa las componentes de la estimación de $x$ dada a la salida de la red; $n(l-1)$ da el número de neuronas en la $(l-1)^{t h}$ capa. La función $f($.$) es la función de activación de la neurona, la$ cual es sigmoidal. Las observaciones deben ser estandarizadas para que caigan dentro de un hipercubo unitario.

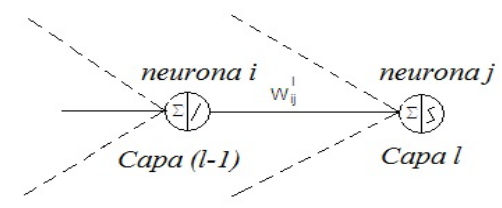

Fig. 2. Conexión de la $i^{t h}$ neurona de la $(l-1)$ capa con la $j^{t h}$ neurona de la $l$ capa

Si la red tiene que aprender una tarea específica, es necesario ajustar los pesos de las conexiones entre las neuronas para minimizar la diferencia entre la salida esperada y la salida dada por la RNAA. Esta minimización es llevada a cabo cuando se calcula el error diferencial. El método más comunmente usado es el de retropropagación.

\subsection{Máquina de soporte vectorial (MSV)}

Sea $x_{i}(i=1, \ldots, M)$ una entrada $n$-dimensional perteneciente a la Clase I o a la Clase II y las etiquetas asociadas $y_{1}=1$ para la Clase I y $y=-1$ para la Clase II. Para datos linealmente separables, puede determinarse un hiperplano $f(x)$ que separe los datos:

$$
f(x)=w \cdot x+b=\sum_{j=1}^{n} w_{j} x_{j}+b .
$$

Un hiperplano de separación satisface las restricciones que definen la separación de los datos muestrales, por ejemplo, $f\left(x_{i}\right) \geq+1$ si $y_{i}=+1$, y $f\left(x_{i}\right) \leq-1$ si $y_{i}=-1$. De esto resulta la ecuación 3:

$$
y_{i} f\left(x_{i}\right)=y_{i}\left(w \cdot x_{i}+b\right) \geq 1, \text { for } i=1, \ldots, M,
$$

donde $w$ es un vector $n$-dimensional y $b$ es un escalar. La notación $w \cdot x_{i}$ corresponde al producto punto de los vectores $w$ y $x_{i}$. El vector de pesos $w$ 
define la dirección del hiperplano de separación $f(x)$ y con $w$ y $b$ (bias) es posible definir la distancia del origen al hiperplano. El hiperplano de separación que tiene la máxima distancia entre el hiperplano y el dato más cercano es llamado hiperplano de separación óptimo. La Figura 3 muestra un ejemplo del hiperplano de separación óptimo de dos conjuntos de datos. Éste es perpendicular a la línea más corta entre las líneas de los límites de los dos conjuntos de datos y el plano, y la línea más corta que intersecta a cada uno a la mitad de la línea.

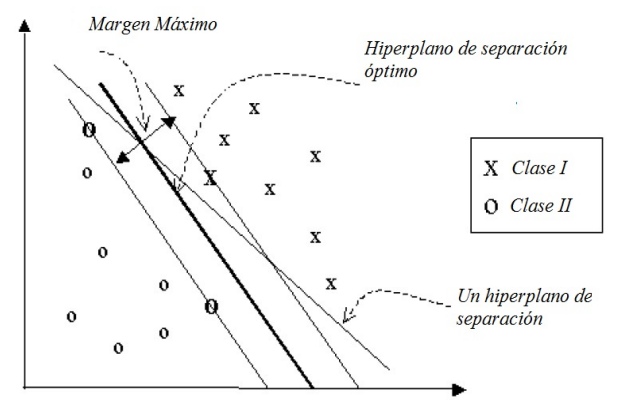

Fig. 3. Hiperplano de separación óptimo

El margen geométrico $\gamma$ es la mitad de la suma de las distancias entre hiperplanos de separación arbitrarios y el dato negativo y positivo más próximo $\left(x^{-} \mathrm{y} x^{+}\right)$:

$$
\gamma=\frac{1}{2}\left[\left(\frac{w}{\|w\|_{2}} \cdot x^{+}\right)-\left(\frac{w}{\|w\|_{2}} \cdot x^{-}\right)\right]=\frac{1}{2\|w\|_{2}}\left[\left(w \cdot x^{+}\right)-\left(w \cdot x^{-}\right)\right]=\frac{1}{\|w\|_{2}} .
$$

Sin perder la generalidad el hiperplano de separación óptimo puede buscarse empleando las llamadas ecuaciones canónicas de hiperplanos, las cuales satisfacen $w \cdot x^{+}+b=1$ y $w \cdot x^{-}+b=-1$. El hiperplano óptimo maximiza el margen geométrico. Así el hiperplano óptimo puede encontrarse resolviendo el problema de optimización cuadrático presentado en la ecuación 5:

$$
\text { minimizar } \frac{1}{2}\|w\|^{2} \text { sujeto a } y_{i}\left(w \cdot x_{i}+b\right) \geq 1 \text {. }
$$

La ecuación 5 puede ser simplificada considerablemente al convertir el problema con las condiciones de Karush-Kuhn-Tucker (KKT) al problema dual equivalente de Lagrange, obteniendo la función de decisión mostrada en la ecuación 6 :

$$
f(x)=\sum_{i=1}^{M} \alpha_{i}^{*} y_{i} x_{i} \cdot x+b^{*} .
$$

Entonces el dato muestral desconocido $x$ puede ser clasificado como Clase I si $f(x)>0$ o como Clase II en caso contrario. 
La MSV también puede aplicarse a casos donde los datos no sean linealmente separables. De esta manera la MSV mapea el vector de entrada en una dimensión espacial mayor. Este proceso esta basado en la selección de una función kernel. Algunas de las funciones kernel más empleadas son: lineales, polinomiales, de base radial y sigmoides. Adicionalmente entre los métodos mayormente aplicados para lograr una clasificación multiclase con la MSV está el conocido uno contra todos el cual construye $M$ MSV donde $M$ es igual al número de clases a ser clasificadas, ya que cada una de estas MSV separa una clase del resto. Otro método es conocido como uno contra uno $M *(M-1) / 2$. Esta MSV combina su función de clasificación para determinar la clase a la cual la muestra de prueba pertenece mediante la acumulación de votos. La predicción con la mayoría de los votos proporciona la clasificación final.

\section{Descripción de la propuesta}

La presente propuesta es una metodología basada en el historial del proceso. Se necesitan bases de datos en modo de operación normal del sistema para entrenar una RNAA y llevar a cabo el proceso de detección. También son necesarias bases de datos que contengan información de las diferentes fallas que se pudieran presentar en el sistema que se monitorea. Con éstas bases de datos de las posibles fallas se da aprendizaje a la MSV multiclase, la cual es la encargada de dar el diagnóstico final cuando una fallas está presente. El proceso general de entrenamiento del sistema de detección y diagnóstico propuesto se muestra en la Figura 4.

El algoritmo para el proceso de entrenamiento se resume de la siguiente manera:

1. Localizar las mediciones provenientes de los sensores de las variables que se desean monitorear.

2. De manera aleatoria tomar un subconjunto $(\approx 80 \%)$ de la cantidad total de datos para entrenar las herramientas de softcomputing empleadas por la propuesta en la primera y segunda fase.

Para la Priemera Fase

3. Tomar bases de datos de la operación normal del sistema.

4. Estandarizar el subconjunto de datos. Entrenar la RNAA y aprender el modelo.

5. Obtener los residuos de las condiciones de operación normal y sus correspondientes límites (umbrales).

Para la Segunda Fase

6. Tomar bases de datos de las diferentes fallas que pudieran presentarse en el sistema.

7. Estandarizar el subconjunto de datos. Entrenar la MSV multiclase y aprender el modelo.

8. La MSV multiclase arroja el diagnóstico final.

Durante la primera fase del diagnóstico, el proceso de detección se lleva a cabo al obtener los residuos generados por la RNAA. Antes que todo, se debe 


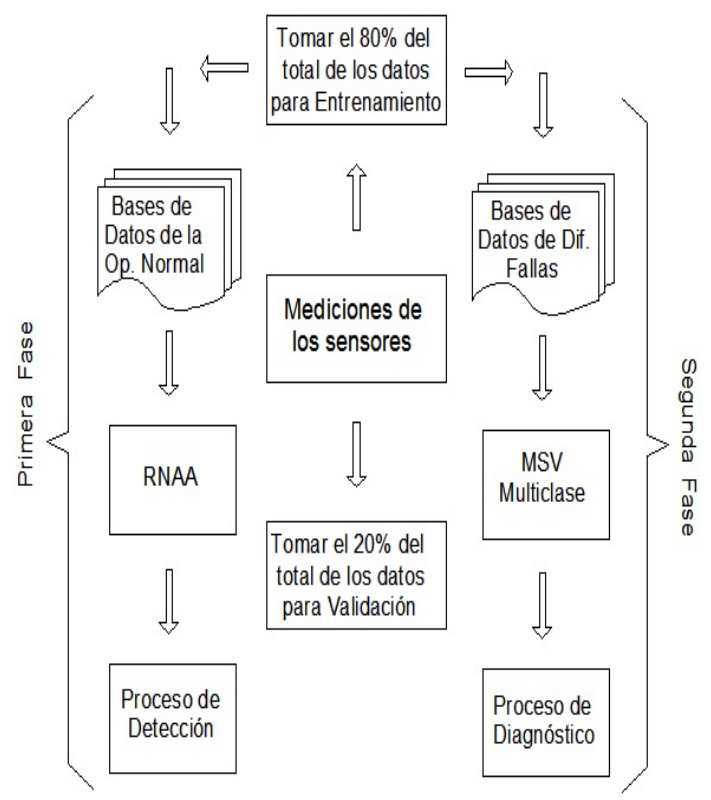

Fig. 4. Proceso general de entrenamiento del sistema de detección y diagnóstico propuesto

de realizar un proceso de estandarización para poder manipular las diferentes variables que se monitorean sobre una misma escala. Posteriormente se genera aleatoriamente un subconjunto de muestras formado por el $80 \%$ del total de la base de datos. Dicho subconjunto de muestras es aprendido por la RNAA. Una RNAA de cinco capas es una red cuyas salidas son entrenadas para emular las entradas sobre un adecuado rango dinámico. Esta característica de la red es muy importante para monitorear variables de sistemas complejos que presentan un cierto grado de correlación entre ellas puesto que cada salida recibe información de cada una de las entradas. Durante el entrenamiento, para hacer que cada salida iguale a su correspondiente entrada, las interrelaciones entre todas las variables de entrada y cada salida individual se refleja en los pesos de conexión de la red. Como resultado de lo anterior se tiene que cada salida específica e incluso la correspondiente salida refleja solo una pequeña fracción del cambio de la entrada sobre un rango de valores razonablemente amplio. Ésto permite a la RNAA detectar la presencia de una falla al comparar simplemente cada una de las entradas con su correspondiente salida, obteniendo de esta manera los residuos. Posteriormente se calculan los límites de dichos residuos para condiciones de operación normal del sistema.

Una vez que el proceso de detección es llevado a cabo, la segunda fase de la propuesta empieza a trabajar. Ésta utiliza la MSV multiclase para clasificar la falla presente previamente detectada por la primera fase. La salida de la MSV multiclase indica cual variable se encuentra en modo de falla y el tiempo en 
el cual dicha falla ha ocurrido, proporcionando de esta manera un diagnóstico completo del sistema que se monitorea. La Figura 5 muestra el esquema de operación en línea del sistema de diagnóstico propuesto.

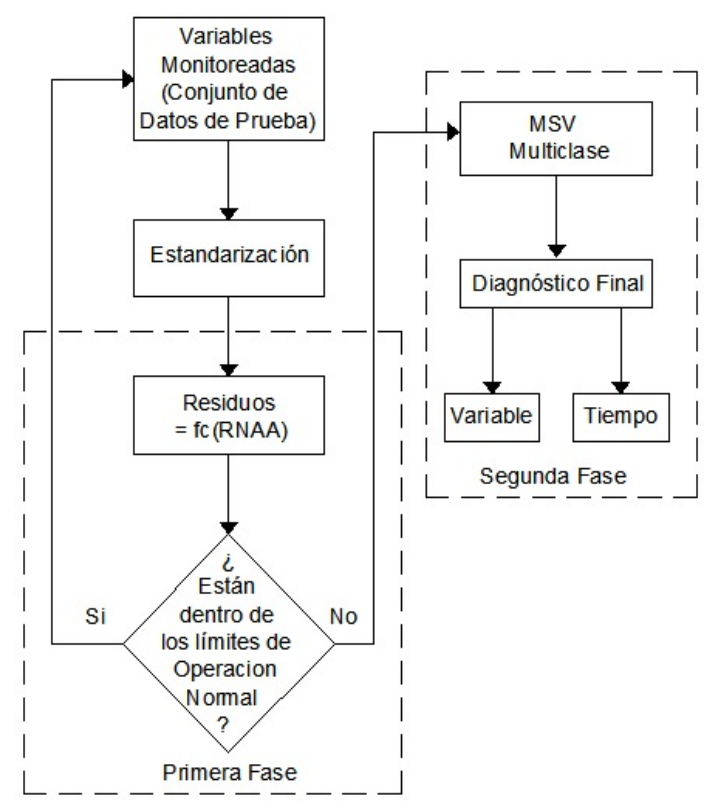

Fig. 5. Esquema de operación en línea del sistema de diagnóstico propuesto

\section{Caso de estudio}

La presente propuesta se validó y se puso en marcha en el Laboratorio de Manufactura de la Facultad de Ingeniería de la Universidad Autónoma de Coahuila. El cual cuenta con una celda de manufactura automatizada (CMA). Se hace mención que la CMA representa un sistema conformado por equipos de alta tecnología, en el que dichos equipos y componentes están integrados para realizar un proceso de manufactura completamente automatizado. Por lo anterior se tiene la necesidad de contar con un sistema de detección y diagnóstico de fallas que lleve a cabo un monitoreo de los equipos más susceptibles a la presencia de éstas y que intervienen dentro de un proceso en la CMA para evitar dejar fuera de operación a la misma. Es por lo anterior que la CMA representa un escenario complejo, perfecto para implementar el sistema de monitoreo, detección y diagnóstico de fallas en su banda transportadora de material. 


\subsection{Posibles fallas que se pueden presentar en el equipo monitoreado}

La CMA cuenta con una banda transportadora para el traslado de pallets. Dichos pallets los toma un robot y los deposita en la banda transportadora. Una vez en la banda, ésta los transporta hacia su otro extremo en donde se emula un proceso de manufactura. Es por ello que se decide que el equipo a monitorear es la banda transportadora de material por representar un equipo que realiza una operación crítica dentro de la CMA así como dentro de un proceso de manufactura real. Debido a que el sistema de diagnóstico está basado en el historial del proceso, es que se puede monitorear todo tipo de variables ya que éstas son tratadas por la metodología solamente como un conjunto de números, sin importar si éstos son provenientes de un sensor de temperatura, presión, voltaje, frecuencia, corriente, etc. Lo cual dota de versatilidad a la metodología propuesta.

El sistema de la banda transportadora de material de la CMA está compuesto por 2 motores eléctricos de inducción trifásicos con rotor tipo jaula de ardilla.

Ya que dichos motores tienen una alimentación trifásica, se decide monitorear el voltaje de cada una de las líneas de alimentación para cada motor. Se considera que el problema de diagnóstico y detección de fallas de la banda transportadora representa una tarea retadora ya que como se comentó, las variables observadas serán los voltajes de alimentación de cada motor y como se pudo constatar en la revisión del estado del arte de sistemas eléctricos, se tiene que el monitoreo y diagnóstico de un sistema eléctrico no es una tarea trivial dada la presencia de características no lineales y presencia de ruido en la medición de las variables involucradas. En los motores eléctricos existe una gran cantidad de perturbaciones tanto internas como externas que afectan su funcionamiento originando de esta manera la presencia de fallas. Dentro del amplio espectro de condiciones que afectan a los motores eléctricos, el presente análisis se centra sobre las fallas que involucran el voltaje de alimentación de cada uno de los motores. Dichas fallas pueden ser fallas simétricas o fallas asimétricas.

1. Las fallas asimétricas se presentan cuando una, dos o las tres líneas de alimentación eléctrica quedan unidas al punto de referencia conocido como tierra.

2. Las fallas simétricas se presentan cuando dos de las léneas de alimentación eléctrica quedan unidas entre sí. Es decir, la magnitud de dos de las tres líneas disminuye y adicionalmente dichas líneas se colocan en fase.

El sistema de diagnóstico propuesto se adecuó al caso de estudio como se muestra en el esquema de la Figura 6 y se describe como sigue.

El desempeño de la metodología para el diagnóstico de múltiples fallas fue evaluado con 50 escenarios. Tales escenarios contenían fallas simétricas y asimétricas incluidas de manera aleatoria en los diferentes motores y sus respectivas líneas de alimentación eléctrica. Se realizó la adquisición y generación de bases de datos mediante la adquisición de ventanas de datos compuestas con 100 muestras por ser la forma sugerida de llevarlo a cabo de acuerdo con la revisión del estado del arte. Se consideraron tres posibles casos de información muestreada. 
Detección y diagnóstico de fallas en sistemas complejos de manufactura ...

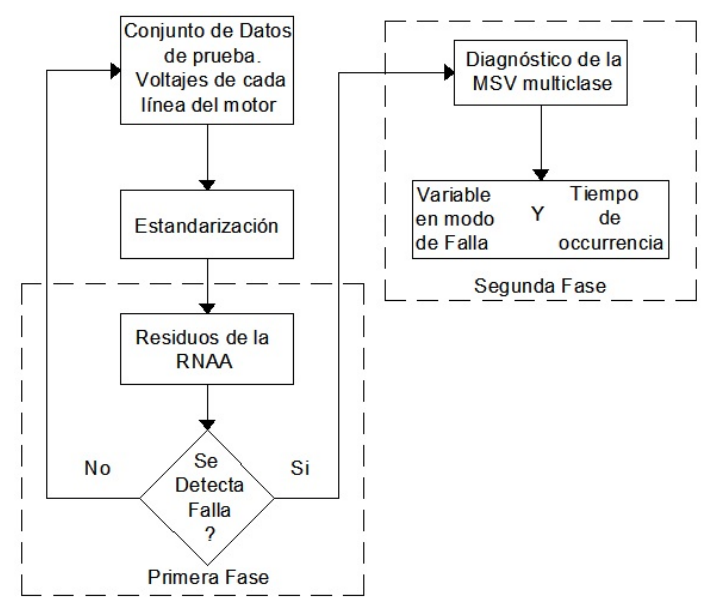

Fig. 6. Implementación de la metodología propuesta en el caso de estudio

- Caso 1: El sistema opera correctamente durante 25 muestras y en modo de falla durante 75 muestras.

- Caso 2: El sistema opera en modo normal durante 50 mestras y las 50 restantes en modo de falla.

- Caso 3: El sistema opera correctamente las primeras 75 muestras y las restantes 25 en modo de falla.

Falla simétrica. Se muestra como ejemplo la inducción de una falla simétrica entre las líneas $L A$ y $L C$ dentro de las primeras 25 muestras de la ventana del conjunto de datos de prueba. De acuerdo a la Figura 6 los datos de prueba se estandarizan y la primera fase de la metodología comienza a trabajar. La salida de la RNAA se muestra en la Figura 7 inciso $(a)$. En ella se observa que la RNAA detecta la presencia de una falla puesto que existen datos que caen fuera del espacio de operación normal obtenido. Posteriormente comienza a trabajar la segunda fase de la metodología, en donde la MSV multiclase arroja el diagnóstico final indicando el tipo de falla presente y el tiempo de ocurrencia, lo cual se muestra en el inciso (b) de la Figura 7.

Falla asimétrica. También se realizó la inducción de una falla asimétrica, la cual fue líneas $L A$ y $L B$ a tierra a partir de la muestra 51 de la ventana del conjunto de datos de prueba. Los datos de prueba se estandarizan y la primera fase de la metodología comienza a trabajar. La salida de la RNAA se muestra en la Figura 8 inciso $(a)$. En ella se observa que la RNAA detecta la presencia de una falla puesto que existen datos que caen fuera del espacio de operación normal obtenido. Posteriormente en la segunda fase de la metodología la MSV multiclase arroja el diagnóstico final indicando el tipo de falla presente y el tiempo de ocurrencia, lo cual se muestra en el inciso $(b)$ de la Figura 8.

Las Tablas 1, 2 y 3 muestran el desempeño del sistema de diagnóstico de fallas para los tres casos respectivamente al considerar diferentes escenarios. Los 


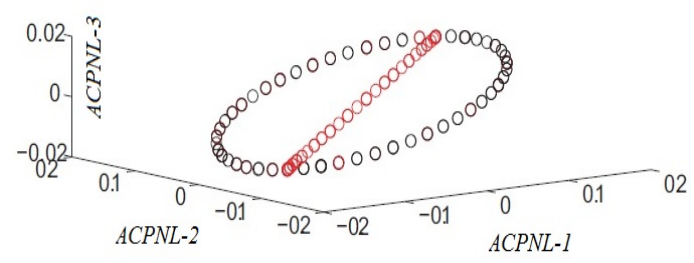

b) Segunda Fase: MSV multiclase diagnóstica el tipo de falla y el tiempo de ocurrencia

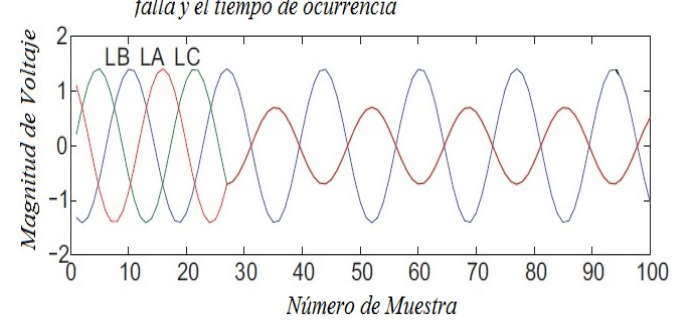

Fig. 7. Falla simétrica Caso 1

porcentajes mostrados corresponden a 50 diferentes escenarios para cada caso y la combinación de diferentes tipos de falla. La primer columna muestra el tipo de falla inducida en los motores. 1 es una línea a tierra, 2 son dos líneas a tierra, 3 son tres líneas a tierra, 4 y 5 representan falla entre dos líneas. La segunda columna da el porcentaje de detección de la falla. La tercera columna es el porcentaje de identificación de manera correcta del tipo de falla presente en el sistema. La cuarta columna muestra el porcentaje de la ubicación correcta del motor en modo de falla. La columna de precisión da el porcentaje de veces que el sistema realizó un diagnóstico correcto.

Tabla 1. Porcentajes de desempeño del sistema de diagnóstico para el Caso 1

\begin{tabular}{|c||c||c||c|c|}
\hline Tipo de Falla Presente & Detección & Identificación & Localización & Precisión \\
\hline 1 & 100 & 100 & 100 & 100 \\
\hline 2 & 100 & 100 & 100 & 100 \\
\hline 3 & 100 & 100 & 100 & 100 \\
\hline 4 & 90 & 90 & 90 & 90 \\
\hline 5 & 90 & 90 & 90 & 90 \\
\hline
\end{tabular}


Detección y diagnóstico de fallas en sistemas complejos de manufactura ... a) Primera Fase: RNAA detecta falla
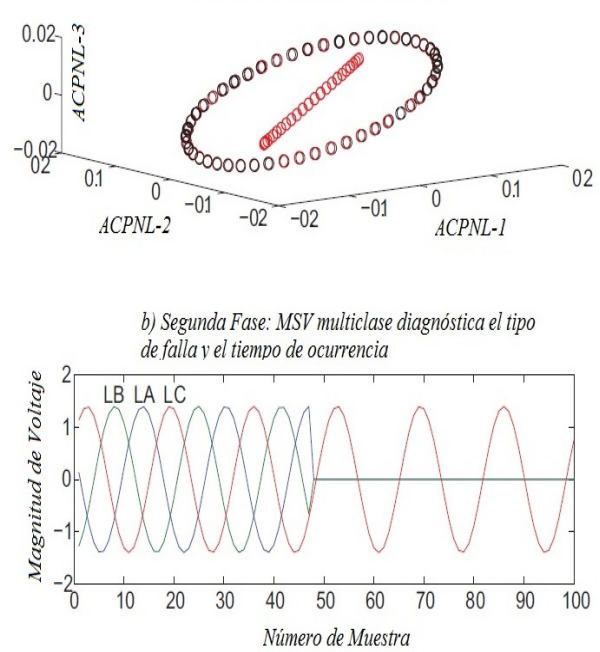

Fig. 8. Falla asimétrica Caso 2

Tabla 2. Porcentajes de desempeño del sistema de diagnóstico para el Caso 2

\begin{tabular}{|c||c||c||c|c|}
\hline Tipo de Falla Presente & Detección & Identificación & Localización & Precisión \\
\hline 1 & 100 & 100 & 100 & 100 \\
\hline 2 & 100 & 100 & 100 & 100 \\
\hline 3 & 100 & 100 & 100 & 100 \\
\hline 4 & 92 & 92 & 92 & 92 \\
\hline 5 & 92 & 92 & 92 & 92 \\
\hline
\end{tabular}

Tabla 3. Porcentajes de desempeño del sistema de diagnóstico para el Caso 3

\begin{tabular}{|c||c||c||c|c|}
\hline Tipo de Falla Presente & Detección & Identificación & Localización & Precisión \\
\hline 1 & 100 & 100 & 100 & 100 \\
\hline 2 & 100 & 100 & 100 & 100 \\
\hline 3 & 100 & 100 & 100 & 100 \\
\hline 4 & 95 & 92 & 92 & 93 \\
\hline 5 & 95 & 92 & 92 & 93 \\
\hline
\end{tabular}

\section{Conclusiones}

Se presentó una metodología para llevar a cabo un diagnóstico completo de sistemas complejos. La propuesta se basa en el análisis y manejo de los datos históricos del proceso. El diagnóstico final es llevado a cabo en dos fases. La 
primera fase emplea una red neuronal autoasociativa para obtener los residuos entre el modo de operación normal previamente aprendido y la información de las mediciones provenientes de los sensores. Una vez que la primera fase detecta la presencia de una falla, la segunda fase comienza a trabajar para dar el diagnóstico final. La segunda fase utiliza una máquina de soporte vectorial multiclase, la cual clasifica la falla presente y proporciona su tiempo de ocurrencia.La metodología propuesta disminuye el problema de falsas alarmas debido a que toma en cuenta la correlación entre las variables monitoreadas mediante la RNAA.

\section{Referencias}

1. Z. Liao, F. Wen, W. Guo, X. He, W. Jiang, T. Dong, J. Liang, B. Xu: An Analytical Model and Otpimization Technique Based Methods For Fault Diagnosis in Power Systems. In: IEEE Third International Conference on Electric Utility Deregulation and Restructuring and Power Technologies, Nanjing, China, pp. 1388-1393 (2008)

2. R.A. Flauzino, V. Ziolkowski, I.N. da Silva, D.M.B. de Souza: Hybrid Intelligent Architecture for Fault Identification in Power Distribution Systems. In: PES'09, IEEE Power \& Energy Society General Meeting, pp. 1-6 (2009)

3. F. Fahimi, D. Brown, M. Khalid: Feature Set Evaluation and Fusion for Motor Fault Diagnosis. In: IEEE Symposium on Industrial Electronics and Applications (ISIEA 2010), Penang, Malaysia, pp. 634-639 (2010)

4. I.Aydin. M. Karakose, E. Akin: FPGA Based Real Time Fuzzy Fault Detection Algorithm. In: IEEE International Conference on Soft Computing and Pattern Recognition (SoCPaR), pp. 389-394.

5. J.P. Nieto, L.E. Garza, R. Morales: Multiple Fault Diagnosis in Electrical Power Systems with Dynamic Load Changes Using Probabilistic Neural Networks. Computación y Sistemas, Vol. 14, No. 1, pp. 17-30 (2010)

6. K. Vinoth Kumar, S. Suresh Kumar, B. Praveena, J.P. John, J. Eldho Paul: Soft Computing Based Fault Diagnosis. In: IEEE Second International Conference on Computing, Communication and Networking Technologies, pp. 1-7 (2010)

7. L. Yuan, Y. He, J. Huang, Y. Sun: A New Neural Network Based Fault Diagnosis Approach for Analog Circuits by Using Kurtosis and Entropy as a Preprocessor. IEEE Transactions on Instrumentation and Measurement, Vol. 59, No. 3, pp. 586-595 (2010)

8. Q. Alsafasfeh, I. Abdel-Qader, A. Harb: Symmetrical Pattern and PCA Based Framework for Fault Detection and Classification in Power Systems. In: Conference on Electro Information Technology, pp. 1-5 (2010)

9. J.A. Jiang, C.L. Chuang, Y.C. Wang, C.H. Hung, J.Y. Wang, C.H. Lee, Y.T.Hsiao: A Hybrid Framework for Fault Detection, Classification, and Location Part I: Concept, Structure, and Methodology. IEEE Transactions on Power Delivery, Vol.26, No.3, pp. 1988-1998 (2011)

10. N. Qianwen, W. Youyuan: An Augmented Naive Bayesian Power Network Fault Diagnosis Method based on Data Mining. In: Asia-Pacific Power and Energy Engineering Conference (APEEC), pp. 1-4 (2011)

11. R.J. Romero, R. Saucedo, E. Cabal, A. Garca, R. A. Osornio, R.A. Salas, H. Miranda, N. Huber: FPGA-Based Online Detection of Multiple Combined Faults in Induction Motors Through Information Entropy and Fuzzy Inference. IEEE Transactions on Industrial Electronics, pp. 5623-5270, Vol. 58, No. 11 (2011) 
Detección y diagnóstico de fallas en sistemas complejos de manufactura ...

12. J.P. Nieto: Multiple Fault Diagnosis in Electrical Power Systems with Dynamic Load Changes Using Soft Computing. In: 11th Mexican International Conference on Artificial Intelligence, MICAI 2012, San Luis Potosi, Mexico, Advances in Computational Science Proceedings, Part 2, pp. 319-330 (2012)

13. M. Kramer: Nonlinear Principal Component Analysis Using Autoassociative Neural Networks. AIChe Journal, Vol.37, No. 2, pp. 233-234 (1991) 